\title{
The potential role of immunological adjuvants

\author{
in influenza vaccines
}

\author{
DEREK HOBSON \\ M.D., M.R.C.Path. \\ Department of Medical Microbiology, University of Liverpool, Liverpool
}

\begin{abstract}
Summary
Aqueous influenza vaccines seldom induce longpersistent protection against epidemic risk, and often fail to immunize up to one-third of the recipients, especially when there has been no previous immunological experience of the serotype of virus contained in the vaccine. Attempts to improve the situation by increasing the size or number of doses are limited by expense, toxicity and the time available in face of an oncoming epidemic. The future use of isolated viral antigens as vaccines may be limited by similar disadvantages.

Immunological adjuvants may make a valuable contribution in decreasing the minimum effective dose of antigen, thereby reducing the toxicity and expense of the vaccine, and increasing its availability. Adjuvant vaccines used in the past have been shown to induce higher mean titres of circulating virus-neutralizing or $\mathrm{HI}$ antibody in a higher proportion of the population than have aqueous vaccines, and the duration of immunity has been prolonged. The choice of adjuvants and the avoidance of untoward side-effects are discussed in this review.
\end{abstract}

THE best immunological responses to aqueous inactivated influenza virus vaccines usually occur when one needs them least, right at the end of the decade of prevalence of a particular sub-type such as the Asian strains. By this time the viruses are well adapted to laboratory growth, and large-scale production can readily provide a surplus of antigen which may be used freely to increase the number of doses per person, or to increase the size of the dose, especially by means of zonal centrifuge concentration, without limiting the total number of people who can be vaccinated. Furthermore, the vaccine is only required to recall or boost a pre-existing immunity rather than induce a new pattern of response in an immunologically virgin population.

The underlying inefficiency of aqueous vaccines emerges in the vital few months after the abrupt appearance of an antigenically novel influenza $\mathbf{A}$ virus, as in 1957 and 1968, when new vaccines must be made under emergency conditions from an ill- adapted virus. In neither of these pandemics was there any convincing evidence that either the quality or scale of vaccination was enough to control the spread of the disease through any country. Under these circumstances, when most people are absolutely devoid of prior experience, a single dose of conven-

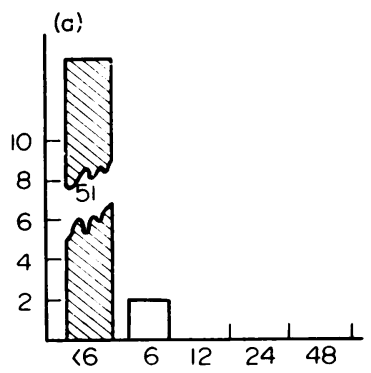

(b)
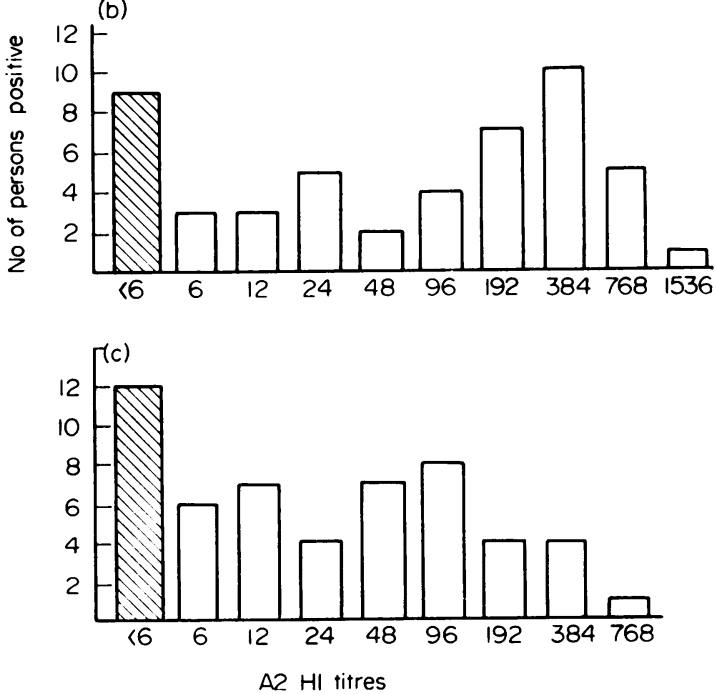

FIG. 1. The response of adult volunteers with no previous experience of Asian influenza to inactivated vaccine (7500 HA units of A2/Sing/1/57, subcutaneous). (Unpublished data, Hobson, 1960.) (a) Prevaccine GMT= $<6$, (b) 4 weeks postvaccine $G M T=49 \cdot 9$, (c) 1 year postvaccine $\mathrm{GMT}=19 \cdot 9$. 
tional vaccine (7500 HA units per dose) tends to give low conversion rates and far too wide a scatter of response (Fig. 1. Hobson (1960) unpublished data); over $20 \%$ of people may be left with no detectable haemagglutination-inhibiting (HI) antibody in the serum, and a further $20 \%$ only acquire titres of very dubious protective value. Moreover, the immune response that is induced often does not persist for even twelve months after vaccine. Vaccinated people may thus be fully susceptible again by the time a second epidemic wave of the same virus serotype occurs. Because of this short duration of immunity, together with the toxic side-effects of many aqueous preparations (Meichen, Rogan \& Howell, 1962) health authorities and large industrial organizations are reluctant ever to initiate mass vaccination campaigns against influenza, since they could be faced with the problem of annual revaccination to maintain the status quo.

The immune response to aqueous vaccines is often worse than after natural infection, not only in terms of strictly homologous serum $\mathrm{HI}$ antibody, but also in the spectrum of serological activity against other influenza A viruses of the same sub-type but with minor degrees of antigenic drift. Brown, Gajdusek \& Morris (1969) found that isolated communities in Micronesia, with no pre-existing exposure to A2

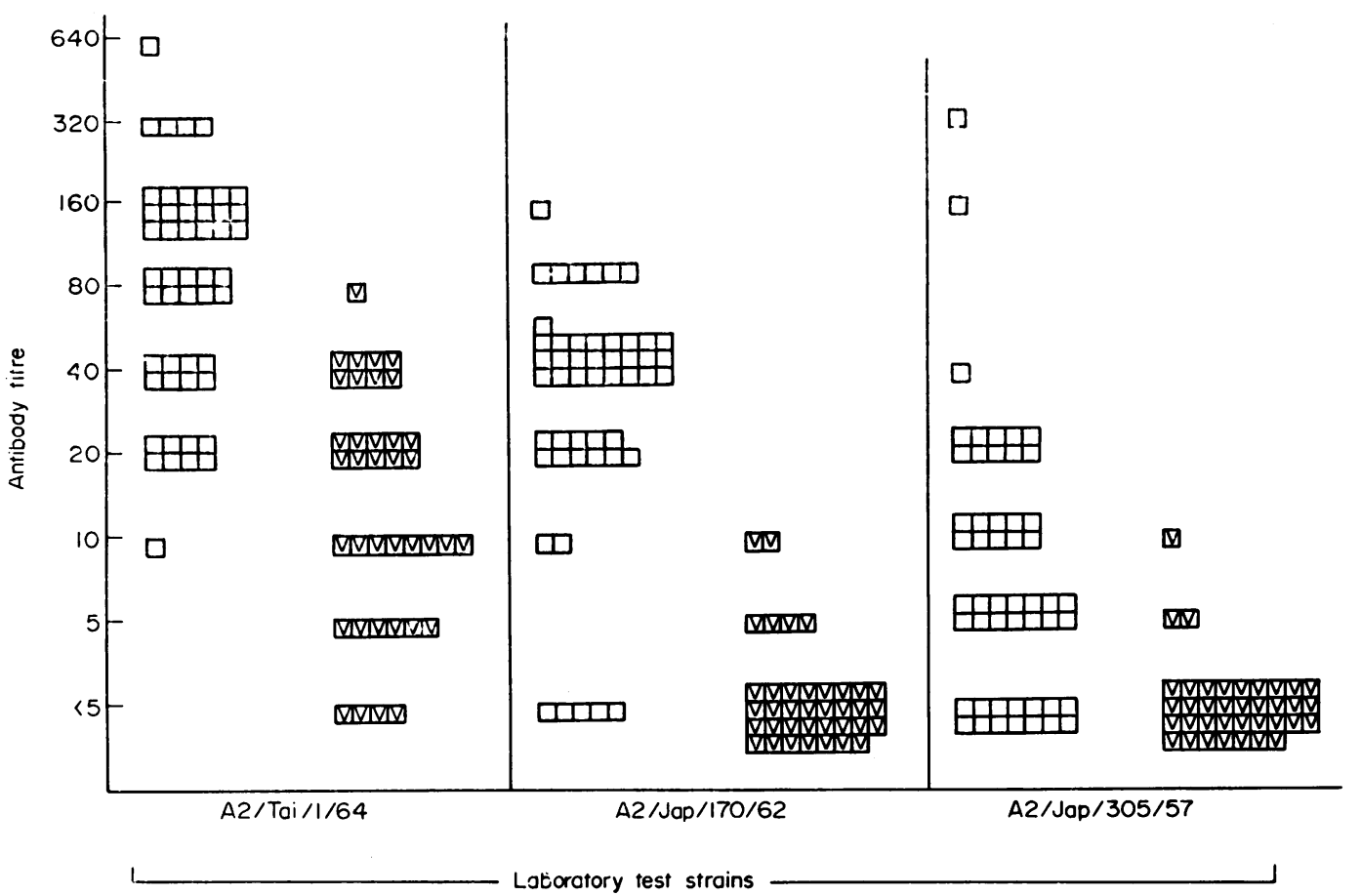

Fig. 2. Differences in the serum antibody response of an immunologically virgin population to vaccination with A2/Tai/1/64 or infection with an antigenically identical strain. (Redrawn from data of Brown et al., 1969.) Open squares, infection; $\mathrm{V}$ in square, vaccine. viruses, developed lower titres of homologous antibody after vaccination with 300 CCA of A2/Tai/1/64 than after natural infection with an antigenicallyidentical strain (Fig. 2) and showed significantly less induction of cross-reacting $\mathrm{HI}$ antibody against the earlier A2 strains of 1957-62. Similarly, Weibel et al. (1967) found that aqueous vaccines of a particular A2 strain confer little serological activity against sequentially later antigenic variants.

These immunological shortcomings of aqueous preparations may well become even more pronounced when separated purified $\mathrm{HA}$ and neuraminidase $(\mathrm{N})$ antigens come into more general use in place of the whole virus particle, with the removal of the inbuilt adjuvant effect of a large carrier rich in lipid. The low efficiency of recovery and the cost and time involved in antigenic separation would make it difficult to compensate for any such loss of immunogenicity by increasing the size or number of doses per patient, especially in a crash programme of vaccination against a new virus serotype.

The need for a reliable artificial immunological adjuvant of influenza vaccines is therefore obvious, and anything which could make a poor antigen good, or a good antigen better is worth exploring. In the past 20 years literally everything from tapioca to tubercle bacilli and from shark oil to sesame seeds 
have been investigated, and from the more recent studies of White (1967) and Gall (1967) one can begin to define the essential common features a successful adjuvant should have.

Firstly, many adjuvants show protein-binding activity and thus act as a depot from which antigen can be slowly released at a rate which will not swamp or evade immunocyte recognition mechanisms, and which does not produce gross toxic effects.

Secondly, most adjuvants have local irritant or surfactant properties which stimulate macrophage and lymphocyte infiltration to the site of injection, increase the rate of antigen adsorption to immunocytes and probably stimulate lysosomal breakdown in the committed plasmocyte.

Thirdly, general stimulation of the reticuloendothelial system may be important; adjuvants such as Corynebacterium parvum, bacterial endotoxins, and oligonucleotides need not necessarily be given at the same site or even at the same time as the antigen to exert an adjuvant effect.

Simple compounds such as aluminium salts which are effective adjuvants of bacterial toxoids seem to be of little value with whole virus vaccines (Himmelweit, 1960), presumably because the 'carrier' function of the alum molecules is obviously impossible with such gross particles. However, this type of adjuvant will deserve re-investigation when isolated smallmolecular antigens of influenza such as crystalline HA (Brand \& Skehel, 1972) become available as vaccines.

The only influenzal adjuvants which have been investigated clinically are all variations of the original depot technique of Freund \& McDermott (1942) omitting tubercle bacilli which are obviously not feasible in human vaccines, refining the oil and emulsifier, and defining the optimal viscosity of the oil-vaccine emulsion (Berlin, 1960). The adjuvant vaccine used most commonly for the last 25 years in clinical trials in the USA (Davenport, 1961) and Britain (Hobson et al., 1964) and more recently in commercial production in Britain has been a thick stable water-in-mineral-oil emulsion (Fig. 3a) with antigen slowly released from the discontinuous phase. Many clinicians and licensing authorities have been worried about the irritant effects and long persistence of these non-metabolizable mineral oils in the tissues, and about the remote possibility of their carcinogenicity. Hence Hilleman and his colleagues (1972) have over the past 8 years developed an alternative thick emulsion vaccine, Adjuvant 65 , in which the base is readily-metabolized peanut oil. Herbert (1967) developed an ingenious modification (Fig. 3b), whereby a thin compound or reversed-phase emulsion is made by redispersing the original thick adjuvant to give an antigen-in-water-in-oil-in-water emulsion which is far easier to dispense and inject, (a)

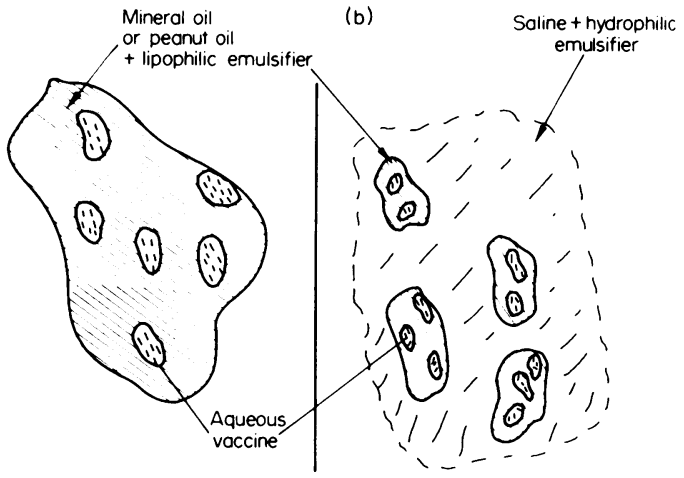

FIG. 3. Oil adjuvant emulsion vaccines. (a) Thick depot emulsion (water-in-oil), (b) reversed phase compound emulsion (water-in-oil-in-water).

less likely to give inflammation or nodulation at the site of injection because it disperses freely throughout the whole RE system, and evokes for this reason an even greater immune response than thick emulsions.

It has long been known that water-in-mineral-oil preparations can very greatly increase not only the percentage of 'antibody-conversions' in vaccinated populations, but also the mean height and duration of the serum HI antibody response. Salk et al. (1953) showed (Fig. 4) that the peak mean titre was twelve times greater after adjuvant vaccine than after an equivalent dose of aqueous vaccine, and 12 months later, $80 \%$ of the adjuvant group had titres of 1024 or greater, compared with only $10 \%$ of the aqueous group. Conversely none of the people given adjuvant vaccine had titres of 64 or less, whereas $30 \%$ of the aqueous group had only low titres of doubtful protective value.

There is no evidence that the slowed release of antigen from depot emulsions delays the onset of immunity in the first weeks after vaccination (Salk

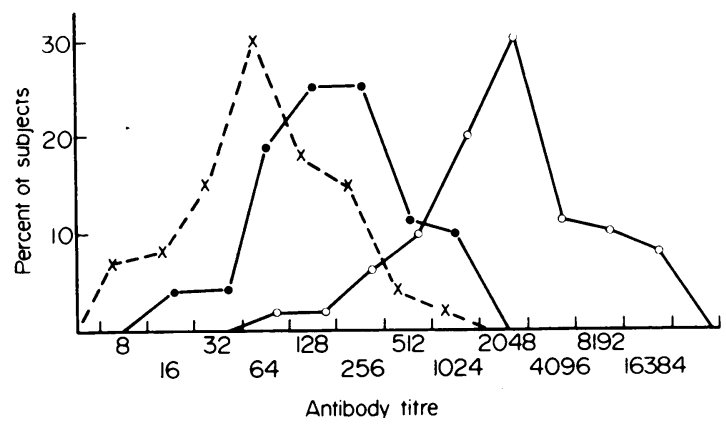

FIG. 4. The distribution of serum HI antibody titres 1 year after aqueous or oil adjuvant vaccines of Ao/PR8 (100 CCA intramuscular). (Redrawn from data of Salk et al., 1953.) $\times---\times$, Prevaccine; - -0 , postaqueous; $\mathrm{O}-\mathrm{O}$, postadjuvant. 
et al., 1953). Moreover, antigenic extinction titrations in volunteers (Fig. 5) show that considerably less virus is required to produce a given rise in antibody when oily adjuvant is used. In subsequent adjuvant vaccine trials with a variety of influenza virus strains serological advantages similar to those described above have been obtained with antigen doses 5-10 times lower than those in conventional aqueous vaccines (Davenport, 1961; Hobson et al., 1964; Herbert, Selwyn \& Philp, 1965).

Thus, one of the greatest potential advantages of immunological adjuvants is to allow a larger number of people to be immunized early after a new epidemic virus has emerged, with vaccine doses small enough to obviate many of the toxic side-effects of aqueous influenza vaccines. Although postvaccine HI titres are usually significantly lower in people with no detectable antibody before adjuvant vaccine than in those with prevaccine experience of the antigen (Hobson, 1967), it is clear that even as a primary immune stimulus a low-dose adjuvant vaccine is better than higher doses in aqueous form. Thus, as shown in Fig. 6, when A/Swine vaccine was given to young children who could not have had prior experience of this antigen (Hennessy \& Davenport, 1961), an initial dose of 60 CCA of adjuvant vaccine gave higher initial HI titres than $200 \mathrm{CCA}$ in aqueous form, and established an effective immunological memory. A second vaccine dose 7 months later gave a good booster response, and although antibody titres subsequently declined the mean level 1 year later was sixteen-fold higher than after aqueous vaccine.

By further manipulation of the size and number of doses, and of the constitution of the adjuvant emulsion it is possible to maintain high $\mathrm{HI}$ titres for at least 7 years after vaccination, and the incorporation of polynucleotides (poly IC) in adjuvant 65 vaccine

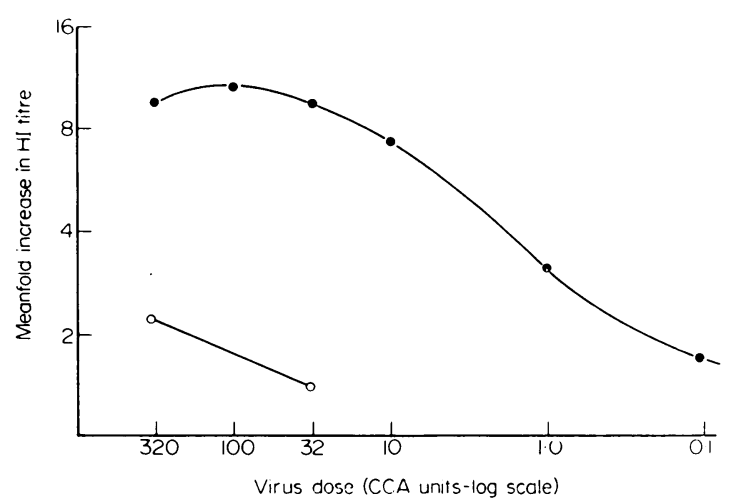

FIG. 5. Antigenic extinction titration of aqueous and adjuvant vaccine of $B /$ Lee (intramuscular) in volunteers. (Redrawn from data of Salk et al., 1953.) O-O, Aqueous vaccine; $\mathbf{0}-\mathbf{O}$, adjuvant vaccine.

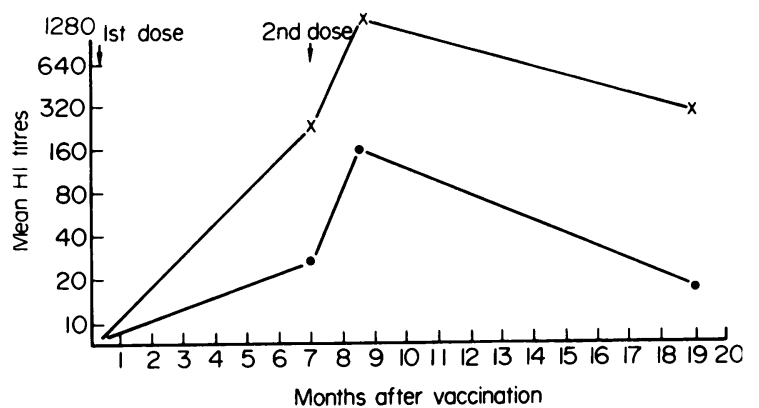

FIG. 6. The serum antibody response to inactivated A/Swine vaccines in children with no previous experience of this antigen. (Redrawn from data of Hennessy \& Davenport, 1961.) $\times-\times$, Adjuvant (60 CCA); - - Aqueous (200 CCA).

gives a further 'synergistic hyper-potentiation' of the immune response (Hilleman et al., 1972). However, it seems doubtful if there is any clearcut advantage in the short term in raising the level of serum HI antibody to a level so many times higher than that needed to protect against homologous infection (Hobson et al., 1972); in the long term, the patient might not only be left with high titres of irrelevant antibody against an obsolete pathogen but the original strenuous process of hyperimmunization might have pre-empted so many immunocytes as to distort or interfere with his response to a subsequent epidemic serotype. The potential advantages of immune hyperpotentiation may perhaps lie more in enabling parenteral vaccines to induce higher titres of secretory IgA antibody, especially against the $\mathrm{N}$ antigen, than is customarily the case (Fedson $e t$ al., 1971) or in inducing antibodies of a wider spectrum of activity. Woodhour et al. (1969) showed that the pre-Hong Kong A2 vaccine strains of 1967 , when given in adjuvant 65 , induced antibody against the Hong Kong virus in $56 \%$ of recipients, whereas this heterologous response occurred only in $2 \%$ of those given the same dosage in aqueous form.

With the undoubted advantages of adjuvant influenza vaccines it is surprising at first sight that they are not already being used on a national commercial scale. The first reason is that it has always been very easy to make a water-in-oil vaccine which does not work. Emulsified vaccines are elegant pharmaceuceutical preparations which require a high degree of skill and care in manufacture, storage and injection if serious untoward effects are to be avoided. The frequency of toxic reactions and nodulation is low, but still unacceptably high for mass vaccination campaigns. Part of the trouble lies with the oils and emulsifiers being insufficiently characterized, but much of the blame falls on the antigen itself-in the past crude whole virus preparations have been used, and the adjuvant will indiscriminately augment 
immune reactions, particularly delayed hypersensitivity reactions, against all the contaminant proteins and lipoproteins as well as against the virus antigens.

Further theoretical disadvantages of adjuvant vaccines still remain to be solved-in animals, it is well known that adjuvants can modify the expression of antigenic determinants, can change the ratio of the various antibody classes and can change the balance between antibody-mediated and cell-mediated immunity (White, 1967). If these events were to happen in man, the possibility of Arthus or delayed hypersensitivity reactions to viral antigens on subsequent vaccination or infection would constitute an unjustified risk, perhaps greater than that of the uncontrolled disease. It must be remembered that epidemic influenza is mainly a socio-economic risk, at the general population level, only once in a decade. To the average individual, influenza is only one of many trivial self-limiting respiratory infections with none of the emotive overtones of poliomyelitis or smallpox. Thus, the safety and purity of influenza vaccines is of paramount importance if they are ever to be accepted by patients on the large scale which alone could control future pandemics.

\section{References}

BerLIN, B.S. (1960) Gross physical properties of emulsified influenza virus vaccines and the adjuvant response. Journal of Immunology, 85, 81.

BRAND, C.M. \& SKeHEL J.J. (1972) A crystalline haemagglutinin subunit of influenza. Nature New Biology, 238, 145.

BROWN, P., GAJDUSEK, D.C. \& MORRIS, J.A. (1969) Antigenic response to influenza virus in man. American Journal of Epidemiology, 90, 327.

DAVENPORT, F.M. (1961) Applied immunology of mineral oil adjuvants. Journal of Allergy, 32, 177.

Fedson, D.S., FulK, R.V., Huber, M.A., Reisberg, M.A. \& KASEL, J.A. (1971) Anti-neuraminidase antibody response in serum and nasal secretions following intranasal or subcutaneous inactivated A2/Hong Kong/68 influenza virus vaccine. Journal of Immunology, 107, 730.

Freund, J. \& McDermotr, K. (1942) Sensitization to horse serum by means of adjuvants. Proceedtings of the Society of Experimental Biology and Medicine, 49, 548.
GALL, D. (1967) Observations on the properties of adjuvants. In: Symposia Series in Immunobiological Standardization. 6. Adjuvants of Immunity, p. 39. Karger, Basle.

Hennessy, A.V. \& Davenport, F.M. (1961) Relative merits of aqueous and adjuvant influenza vaccines when used in a two-dose schedule. Public Health Reports, 76, 411.

Herbert, W.J. (1967) Multiple emulsion adjuvants. In: Symposia Series in Immunobiological Standardization. 6. Adjuvants of Immunity, p. 89. Karger, Basle.

Herbert, W.J., Selwyn, S. \& Philp, J.R. (1965) Field trials of adjuvant and saline influenza vaccines. British Journal of Preventive and Social Medicine, 19, 97.

Hilleman, M.R., Woodhour, A., Friedman, A., Weibel, R.E. \& Stokes, J. (1972) The clinical application of Adjuvant 65. Annals of Allergy, 30, 152.

Himmelweit, F. (1960) Serological responses and clinical reactions to influenza virus vaccines. British Medical Journal, 2, 1690.

Hobson, D. (1967) Serological studies on adjuvant influenza vaccines. In: Symposia Series in Immunobiological Standardization. 6. Adjuvants of Immunity, p. 269. Karger, Basle.

Hobson, D., Curry, R.L., Beare, A.S. \& Ward Gardner, A. (1972) The role of serum HI antibody in protection against challenge infection with influenza A2 and B viruses. Journal of Hygiene, 70, 767.

Hobson, D., Lane, C.A., Beare, A.S. \& Chivers, C.P. (1964) Serological studies on adult volunteers inoculated with oil-adjuvant influenza vaccine. British Medical Journal, 2, 271.

Meichen, F.W., Rogan, E. \& Howell, R.W. (1962) A study $\oslash \vec{\bullet}$ of the effectiveness of influenza vaccination in an industrialcommunity. British Journal of Industrial Medicine, 19, 203.

Salk, J.E., Contakos, M., Laurent, A.M., Sorensen, M., RAPALSKI, A.J., Simmons, I.H.\& SANDBERG, H. (1953) Use? of adjuvants in studies on influenza immunization. Journal of the American Medical Association, 151, 1169.

Weibel, R.E., Woodhour, A.F., Stokes, J., Metzgar, D.P. \& Hilleman, M.R.(1967) New metabolizable immunologic adjuvant for human use. New England Journal of Medicine, 276, 78.

WhITE, R.G. (1967) Concepts relating to the mode of action of adjuvants. In: Symposia Series in Immunobiological Standardization. 6. Adjuvants of Immunity, p. 3. Karger, Basle.

Woodhour, A.F., McAleer, W.J., Friedman, A., Weibel, R.E., Stokes, J. \& Hilleman, M.R. (1969) 1967 formula influenza vaccine in Adjuvant 65. Proceedings of the Society of Experimental Biology and Medicine, 131, 501. 